\title{
Ecological-Enactive Scientific Cognition: Modeling and Material Engagement
}

This is a post-peer-review, pre-copyedit version of an article published in Phenomenology and the Cognitive Sciences. The final authenticated version is available online at: https://link.springer.com/article/10.1007/s11097-020-09713-y

Giovanni Rolla (UFBA) Felipe Novaes (PUC-Rio)

\begin{abstract}
Ecological-enactive approaches to cognition aim to explain cognition in terms of the dynamic coupling between agent and environment. Accordingly, cognition of one's immediate environment (which is sometimes labeled "basic" cognition) depends on enaction and the picking up of affordances. However, ecological-enactive views supposedly fail to account for what is sometimes called "higher" cognition, i.e., cognition about potentially absent targets, which therefore can only be explained by postulating representational content. This challenge levelled against ecological-enactive approaches highlights a putative explanatory gap between basic and higher cognition. In this paper, we examine scientific cognition - a paradigmatic case of higher cognition - and argue that it shares fundamental features with basic cognition, for enaction and affordance selection are central to the scientific enterprise. Our argument focuses on modeling, and on how models promote scientific understanding. We base our argument on a non-representational account of scientific understanding and on the material engagement theory, for models are hereby conceived as material objects designed for scientific engagements. Having done so, we conclude that the explanatory gap is significantly less threatening to the ecological-enactive approach than it might appear.
\end{abstract}

keywords: enactivism; ecological psychology; scientific models; scientific understanding; epistemic artifacts; material engagement.

\section{Introduction}

Enactivism and ecological psychology are two of the main branches of the research tradition on embodied cognition and, despite being historically independent from each other, they are broadly convergent and similar in spirit (Heras-Escribano, 2019). In particular, both enactive and ecological approaches attempt to explain cognition from the bottom-up, focusing on how organisms engage with their immediate environment. Fundamentally, they agree that cognition cannot be fully understood in abstraction from the cognizing organism's bodily morphology 
and its activities in its medium. Against cognitivist views, ecological psychologists emphasize (Bruineberg, Chemero, \& Rietveld, 2019; Chemero, 2009; Rietveld \& Kiverstein, 2014; Turvey, Shaw, Reed, \& Mace, 1981), and some enactivists agree (Barandiaran, 2017; Di Paolo et al., 2017; Hutto \& Myin, 2013, 2017)1, that cognition is not fundamentally a matter of representing an external environment. Although there seems to be no agreement on what representations are (Rowlands, 2017), what both these views reject is the need to postulate mental entities with accuracy conditions in order to explain all sorts of cognitive activity. ${ }^{2}$ Instead, for both views, cognitive events take place as an organism explores environmental structures through sensorimotor engagements. In Gibson's foundational work (2015), environmental structures that specify possibilities for action for a given organism are called affordances. Affordance selection occurs as an organism explores these possibilities in effective engagements. According to what is sometimes referred to as autopoietic enactivism, enaction is the selection and refinement of patterns of sensorimotor engagement, which consists in making sense of one's environment, i.e., adaptively exploring points of interest for the organism (Di Paolo, 2005). Assuming that both enactivism and ecological psychology are broadly convergent, in this paper we will treat them as a unified ecological-enactive (henceforth, EE) approach ${ }^{3,4}$. Explanations of the EE kind, thus, aim to account for the cognition of an organism's immediate environment, which is usually labelled "basic" cognition.

Since its early days, embodied accounts of cognition face a compelling objection, namely, that they supposedly fail to scale up and explain the occurrence of "higher" cognition (Edelman, 2003). The problem involves providing an explanation, in embodied terms, of representational-hungry cognitive tasks, such as remembering, inferring, planning and so on-

\footnotetext{
${ }^{1}$ As Hutto and Myin (2013) argue, some enactivists endorse the need of action-oriented representations in their framework, such as Clark (2016). In this paper, however, we will construe 'enactivism' in Hutto and Myin's sense of radical enactivism, i.e., enactivism that rejects the need of any kind of representational content in order to explain basic cognition. For a more fine-grained distinction about varieties of enactivism, see Ward et al., (2017). ${ }^{2}$ Consequently, the non-representationalism of ecological and enactive approaches is an epistemological thesisthey claim that representations are not needed to explain all cognition. This is a weaker claim than the ontological one that says that mental representations do not exist. This is clear, for instance, in Chemero's (2009) work, but not as clear in the arguments against mental representations put forth by radical enactivists (Hutto \& Myin, 2013, 2017).

${ }^{3}$ Others have used the names 'ecological-enactive' (Kiverstein \& Rietveld, 2018; Segundo-Ortin, 2020; van den Herik, 2018, 2020) and 'enactive-ecological' (Carvalho \& Rolla, 2020) in order to refer to the same core ideas as we do here. However, our conception of the EE approach does not rely specifically on those authors' characterizations, but on the one we present above.

${ }^{4}$ See Heft (2020) for a dissenting opinion. We refer to Heras-Escribano (2019) for a thoroughgoing attempt to combine both views.
} 
tasks that do not involve the immediate environment because they require dealing with potentially absent targets (Clark \& Toribio, 1994). The issue is presented as an explanatory gap, and it is specially pressing for EE views due to the fact that they deny that cognition necessarily involves mental representations, which otherwise might seem as the best candidates for fulfilling the role of "stand-ins" for absent targets.

There have been ingenious attempts to bridge the explanatory gap (hereafter, simply 'the gap'). Some aim to explain the emergence of higher cognition from basic cognition and a relaxed variety of naturalism (Hutto \& Myin, 2017; Hutto \& Satne, 2015). Others aim to reconceive the putative representational hunger of some tasks, such as remembering and imagining, so as to recast them in ecological terms (Bruineberg et al., 2019) or in ecological-enactive terms (Kiverstein \& Rietveld, 2018). In this paper, however, we will not follow either line of reasoning. Our main goal is to show that scientific cognition, which is a prime example of higher cognition, can be at least partially explained in the same way as the EE approach explains basic cognition. Our central claim is that scientific cognition crucially involves enaction and affordances selection, and for that we focus on how scientific models are used in order to provide understanding of their targets. There is of course more to scientific cognition than enaction and the picking-up of affordances - for science typically involves explanations, complex uses of language, very sophisticated inferences and so on. Accordingly, we argue that there is a continuum between basic and higher cognition, even if more is involved at the higher end of the cognitive spectrum. In doing so, we aim to show that the gap is significantly less threatening than it otherwise might appear for the EE approach.

This paper is structured as follows. In section 2, we develop the idea of scientific cognition and focus on model-based approaches. We argue that models can be conceived of as epistemic artifacts or as material objects for scientific engagements (Knuuttila, 2005; Knuuttila \& Merz, 2009; Knuuttila \& Voutilainen, 2003). Accordingly, models scaffold inferences about a given domain of inquiry ${ }^{5}$. In arguing for that claim, we rely on a non-representationalist view of models (de Oliveira, 2018) in conjunction with an inferentialist view of scientific understanding (Kuorikoski \& Ylikoski, 2015). In section 3, we explore the idea that model

\footnotetext{
${ }^{5}$ Throughout this text, we say that models 'scaffold' performances of scientific cognition (in model-based science, that is) to emphasize that this kind of performance depends on and is modified by artifacts, which is different from taking models to be external devices that extend internal capacities. We do so in order to differentiate our view from the extended mind hypothesis applied to modeling (the latter view is developed by Kuorikoski and Ylikoski, 2015), as we discuss in 3.1 .
} 
based-cognition is a kind of material engagement, in accordance with the material engagement theory (Malafouris, 2013, 2019). Models are thus understood as materials designed to scaffold our access to their target systems. In section 4 , we conclude by showing that our view on scientific cognition stablishes a continuum between basic and higher cognition. What is novel about our approach to the issue of the explanatory gap is the combining of views and discussions that, despite being well stablished subject matters in their respective fields and offering an interesting intersection, were hitherto kept mostly apart.

\section{Scientific cognition: enaction, affordances and modeling}

\subsection{From explanations to understanding}

Throughout most of its history, philosophy of science has focused on criteria for scientific explanations, usually assuming that explanations are the central, or even the only relevant part of the scientific inquiry. Recently, the concept of understanding has received renewed attention, no longer being construed as a mere epiphenomenon of entertaining correct scientific explanations (for a discussion, see Regt, Leonelli, \& Eigner, 2009). Accordingly, some have argued for a conceptual distinction between understanding and explanation—one can, after all, understand a given phenomenon without being able to explain it (Lipton, 2009). It is now a staple in philosophy of science that the goal of science is to provide understanding about the physical and social world, which can be achieved in different degrees and be done in many different ways. Achieving scientific understanding is what we call "scientific cognition".

Assuming that understanding and explaining are conceptually distinct scientific activities, we examine how model-based science provides understanding about the world, thus putting aside more traditional concerns about the formal constrains on scientific explanations. Furthermore, focusing on understanding is important in our case because we argue that scientific cognition is not radically distinct from basic cognition, and the similarity between the two extremes of this continuum is more clearly attested if we focus on understanding, rather than on explanations. After all, in the EE framework, basic cognition involves the exploration of environmental structures through the picking up of affordances and the selection and refinement of patterns of sensorimotor engagements. As mentioned above, this does not involve representing an external environment, but directly engaging with it. Explanations, on the one hand, are strictly language-based, and - at least to traditional views-language is 
essentially a matter of combining symbols with semantic content, i.e., symbolic representations (for a dissenting opinion, see Di Paolo, Cuffari, \& De Jaegher, 2018; Moyal-Sharrock, 2019). On the other hand, although in scientific practice understanding usually takes place concomitantly with explanations, understanding per se is not representational in nature. And because scientific understanding is typically directed at the so-called target systems through model manipulation, it is therefore directed at potentially absent targets, thus classifying for higher cognition. For this, we will expand on an inferentialist view of understanding, as we will see further below. Before that, however, we must consider the relation between understanding and modeling. Our aim in this section is to characterize model-based science in a way that affords an approximation with the EE account of basic cognition.

\subsection{The materiality of models}

The latter part of the $20^{\text {th }}$ century saw an increased interest on modeling activity in scientific practice, to the extent that nowadays it is widely accepted that models are essentials tools for achieving scientific understanding (Frigg \& Hartmann, 2020). However, many different things have been called 'models', with many qualifications, and sometimes with significant overlapping as well. At this juncture, following Frigg and Hartmann (ibid.), it is important to distinguish between two broad categories of models: a model can be a model of a theory, i.e., it can represent a theory by interpreting its laws and axioms, thus aiming to bridge abstract theoretical entities and experimental data. Alternatively, a model can be a model of a phenomenon, i.e., a simplification of a target system that makes it theoretically tractable. We are interested in the latter notion of model, and we will abstain from making any claims about models of theories.

Models of phenomena include scale models, in which some aspects of the target are kept constant and others are ignored depending on the modeler's purpose. Planetary models, for instance, allow visualization of planetary orbits typically by disregarding the proportional distance between planets. Other kinds of models are analogical models, such as Schelling's checkerboard model of segregation, (see Kuorikoski \& Ylikoski, 2015) and model organisms, such as mice and fruity flies, which are used in biology to study other organisms or to study more general phenomena, such as the effects of crossover between chromosomes (see Leonelli, 2009). Analogical models also include mechanical models, which attempt to capture some mechanical laws governing a given target system through simplification and approximation, 
such as Boltzmann's dumbbell model of diatomic molecules (see Regt, 2009) and the billiard ball model of gases (see Gelfert, 2017). In computer simulation techniques, dynamical and computational models are used, respectively, to specify how a target system evolves over time (typically through non-linear differential equations) and to transform dynamical models into analytically and computationally tractable models. For a discussion of Wilhelmson's computational simulation of a severe thunderstorm, see Winsberg (1999).

There is a strong representational pull in the philosophical literature about the epistemology and ontology of models (see Frigg \& Nguyen, 2017, for an overview). Accordingly, semantic conceptions of models have been the mainstream in philosophy of science (Fraassen, 1980; Giere, 1988; Pincock, 2012). The semantic views in general claim that models are representations of their targets and that it is in virtue of a semantic relation-be it isomorphism or a weaker form of similarity - that they provide us with a positive epistemic status about a target system. However, as Oliveira (2018) makes clear, models are purposefully designed to be unlike their targets in order to make them scientifically tractable. To use Elgin's phrase, models are "felicitous falsehoods" (2007). This in turns threatens a semantic conception of modeling, both when it comes to the ontological claim that models are representations and to the epistemological claim that models provide us with knowledge (or other favorable epistemic statuses) of a target by representing it. For:

If what makes $\mathrm{X}$ a model of $\mathrm{Y}$ is that $\mathrm{X}$ represents $\mathrm{Y}$, then how can $\mathrm{X}$ still be a model of $\mathrm{Y}$ when $\mathrm{X}$ misrepresents $\mathrm{Y}$, or falls short from representing $\mathrm{Y}$ accurately? [...] If, as suggested by [the epistemological claim], models are epistemically valuable in investigations of some target because they represent the target, then how can models sometimes be more epistemically valuable when they misrepresent, i.e., when the representational relationship between the two is faulty? (Oliveira, 2018).

There are, therefore, good reasons to resist the representationalist pull in construing modelbased scientific activity. Some pragmatic approaches to representation (Bailer-Jones, 2003; Giere, 2004; Suárez, 2003), however, try to amend the semantical view by reconceiving scientific representations as a three-place relation, where a model represents a target in virtue of how scientists use models (which includes what scientists believe models represent). As 
Oliveira (2018) also points out, however, pragmatist approaches to representation fare no better than the semantic views, for simply anything can be used to represent anything else. This undermines the epistemological claim that models allow us to gain knowledge (or understanding) about their target by representing them. To be clear, the issue is not with the pragmatism of these accounts; the problem is that they are not pragmatist enough. ${ }^{6}$

Thus, emphasizing the use of models in scientific cognition and giving up on a representationalist account of modeling is a viable alternative to these very well-known problems regarding models and their putative representational character. The work of Tarja Knuuttila (2005) and collaborators paves the way for a more radical pragmatist reaction. ${ }^{7}$ Models are characterized as epistemic artifacts (Knuuttila, 2005; Knuuttila \& Voutilainen, 2003) and as concrete constructed objects (Knuuttila \& Merz, 2009). To say that a model is an epistemic artifact implies

First, that human agency, or rather traces of it, are more or less manifestly present in it. Second, it implies that models are somehow materialized inhabitants of the intersubjective field of human activity. Third, it implies that models can function also as knowledge objects. (Knuuttila \& Voutilainen, 2003, p. 1487)

Models are epistemic artifacts because they are built with the aim of assisting the study of scientific questions - they are what Knuuttila and Merz (2009) call productive rather than representational. The point is made clear on their discussion of parsers, i.e., computational models that offer a syntactic analysis of strings of symbols in order to show the grammatical structure of a language. They argue that parsers are not designed to be realistic depiction of human linguistic competences, because they do not recognize semantic information as we do. Instead, they operate

\footnotetext{
${ }^{6}$ This mirrors the issue of what Hutto and Myin (2013) call "conservative accounts of enactive cognition" (CEC). CEC proponents admit that cognition necessarily involves action, but remain committed to representationalism, albeit of a non-classical kind, one according to which cognition requires action-oriented representations. The problem is that, according to Hutto and Myin (2013), CEC is not pragmatist or radical enough, because mental representations cannot be fully naturalized.

${ }^{7}$ Although Knuuttila (2005) retains a mild representationalism — in her view, models represent due to how they are continuously used and interacted with (see 2005, p. 1269).
} 
By making use of the morphological and syntactic information of the words in the vicinity of the word in question. For instance, the word form "bear" has both nominal and verbal readings; thus, if it is preceded by an article, the parser assigns it a nominal reading. (Knuuttila \& Merz, 2009, p. 160).

Accordingly, parsers are not models of human linguistic competences - they do not aim to represent how humans use language. Instead, they are models for understanding how language works (ibid., 2009, p. 161). A similar point can be made about models in general, for they are conceived in order to afford scientific engagement with their targets in a controlled, tractable manner, not in order to truthfully represent them.

The idea of models as concrete or material objects, which we agree with, is supported by the fact that models are always materialized in certain media. In fact, the best explanation for their success and ubiquity in modern science is that it is precisely in virtue of their inherent materiality that models scaffold scientists to perform certain cognitive tasks more easily. On that view, models are more or less useful for dealing with specific questions depending on the way they are designed and on the cognitive tasks they aim to facilitate. Of course, as proponents of models as epistemic artifacts are willing to agree, models have a mixed ontology in the sense that they consist in a combination of different materials, media and formats (see Gelfert, 2017). The crucial point here is that, without materiality, models could not be intervened upon in order to generate and propagate scientific understanding.

Although it might look implausible to think that model-based cognition depends on the scientists' morphological features, if one subscribes to a thoroughly pragmatist account of modeling, as we do, it follows that embodiment is in fact crucial. This is so because models are designed for our use, and because models are materialized in objects which we can engage with - models are, therefore essentially shaped by the morphological features of their users. Of course, given that models are shared among various members of the scientific community, they are designed with typical human features in mind, instead of those of particular agents. As Knuuttila and Merz (2009, p. 150) argue, if we learn from manipulating and constructing models, it is because models exhibit specific features that afford them to be worked on by creatures like us. 'Affording' here is read precisely in the ecological sense: they offer specific possibilities of engagement, in this case, scientific engagement. Exploring these possibilities is 
a matter of exercising sensorimotor abilities and exploring sensorimotor regularities. Accordingly, what they call the "material embodiment" (ibid., p. 150) of models is no incidental feature - instead, the way models are designed and elaborated is precisely what makes them useful tools in acquiring and sharing understanding. Different goals, theories and areas of inquiry (to name a few factors) require different material approaches in order to make their target systems scientifically workable.

Naturally, the case for the material embodiment of models is uncontentious for material models (such as scale models and model organisms). But one could argue that some models, especially mathematical and computational ones, are entirely abstract, whereas others, such as thought experiments, are straightforwardly fictional.

Expanding on the latter objection, one could either argue for the fictionality of thought experiments or for the bolder claim that all models are fictional. Regarding the first option, it is clear that thought experiments play a crucial role in the scientific enterprise, and they may as well be conceived of as mental models. For instance, Galileo's thought experiment against the supposition that free-falling bodies with different mass values exhibit different accelerations may be taken to be a model of bodies in free fall. Note, however, that thought experiments by definition cannot scaffold one's cognitive capacities-unless they are expressed in a medium, in which case they become undoubtedly material and are no longer thoughts in the strict sense. So, even though models and thought experiments can have similar functions in scientific practice, either the latter are sui generis models or they are no models at all. As for a fictionalist view of modeling in general, one may reply, as Magnani does (2017, chapter 2), that, if models are fictions, one would be hard-pressed, given naturalistic assumptions, to locate so-called abstract models in the brains of scientists and distributed through the scientific community, for they are hardly a product of a single mind. Hence, models are never fictional in the sense that they would not be realized in any medium (see Magnani, 2017, p. 33). Moreover, as Magnani stresses, models are not intended as works of fictionwhich undermines a claim for a general fictionalism.

Now, considering the objection that at least some models are abstract in the sense that they do not rely on any specific material embodiment, we follow Knuuttila and Merz (2009, p. 151) who point out that 3D renderings, diagrams and symbols on paper or on screen are some of the different kinds of materiality that enable scientists to engage in specific manners with their 
targets and to convey their findings with their community. Again, it takes certain phylogenetic and ontogenetic developments to be able to read what is displayed on a screen or on paper, and the way that models are displayed crucially depends on certain physical structures. Therefore, materiality matters even in the case of so-called abstract models. Computer simulations, for instance, typically require computational procedures to convert differential equations that are typical of dynamical models into discrete algebraic equations in order to generate analytically tractable computational models (Winsberg, 1999). That task cannot be accomplished without a computer with certain memory requirements - we could not, for instance, even being to imagine how to do so with an abacus or a clay piece. Thus, a digital computer is not merely the vehicle through which the computational model is conceived, it is part and parcel of modeling itself. It follows that, regardless of their type, models are always concrete means through which we can engage with their target systems (whether these are natural or social phenomena), even in case of unobservable entities.

The general ideas developed in this subsection is that (i) models are not meant to represent and (ii) models are always materialized. To unpack these claims: models are devised by scientists with specific aims in order to deal with specific problems, so they are epistemic artifacts that afford specific forms of engagement and, when done right, scaffold scientific cognition about their target systems. Consequently, the ways scientists actually use models is ultimately dependent upon human bodily morphology as well as the scientists' skills and training. So, as we will see on the next subsection, the heavy explanatory work on how models provide us with understanding is done without appealing to their alleged representational content, which is an important point to explain away the gap between basic and scientific cognition.

\subsection{Models and understanding: a non-representational inferentialist view}

If even a good scientific model does not represent its target system, it might seem puzzling how modeling can generate, enhance and propagate scientific understanding. This issue is especially acute if one subscribes to the idea that scientific understanding is exclusively a matter of propositional understanding. If scientific understanding is understanding-that or understanding-why things are thus-and-so, it is plausibly factive (for discussions on this issue, see Elgin, 2009; Hills, 2009; Pritchard, 2008). Whatever the verdict is on the factivity of propositional understanding, it seems unavoidable that it would be a representational cognitive state. In fact, we are willing to grant that scientific understanding sometimes involves 
propositional understanding (a representational state). But one can concede that scientific cognition sometimes involves understanding that a target system is thus-and-so, and why it is thus-and-so, whilst acknowledging that scientific cognition also involves, at a more basic level, understanding how the target system works. Accordingly, we distinguish between understanding-how and understanding-that/why, and it is the former, not the latter kind of understanding, that is at the core of scientific cognition.

In order to see that, let us consider again the case of a scale model that allows us to understand how planetary orbits work by misrepresenting the distance between planets in our solar system. As stressed above, misrepresentation in such a case is a matter of design - specifically, it favors interaction and visualization, for an accurate proportional distance would render either the planets too small or the distances between them exceedingly large and consequently unmanageable. And yet, a novice that interacts with the planetary model grasps something about the structure of the solar system (say, she may understand how the phenomenon of epicycle takes place). That is because the novice comes to understand how orbits works by engaging with the model, manipulating its pieces, even tinkering with it (if she is the one who built it). Understanding that a planetary system works in a specific way and why it does so can plausibly be further goals of astronomic inquiry, but one can only do that if one understands how the system works, and this is done by manipulating the model, exploring its affordances and sensorimotor regularities. Similar points apply to other kinds of models. The understanding afforded by analogical models of their targets, for instance, fundamentally depends on grasping how the models work. In the case of model organisms, understanding that genetic phenomena happen in certain ways necessarily depends on understanding how they happen in the model organism. In the case of computer simulations, one can only understand that a given phenomenon occurs in a specific way through a collection of data in a simulation if one understands how the models in which the simulation is based actually work. The distinction between understanding-how and understanding-that/why eases the problem of how models allow us to gain understanding about their targets without representing them. Models fundamentally provide understanding not because they accurately represent their target systems, but because they afford successful engagements with those targets.

It is possible, however, that one successfully engages with a model, and yet falls short of understanding how its target works. This is possible because, as we mentioned at the beginning, scientific understanding is directed at a target system by being mediated through a model (thus 
fitting the criteria for higher cognition). So, something has to bridge the gap between understanding how the model works and understanding how the target system works. This can be done with a an inferentialist account of understanding as developed by Kuorikoski and Ylikoski (2015), but with an important qualification regarding the putative representational character of understanding. Kuorikoski and Ylikoski maintain that 'understanding is constituted by the ability to make correct what-if inferences concerning the phenomenon to be understood', an idea they explain as the 'capability to put one's knowledge to use' (ibid.). Accordingly, a good scientific model affords not only the manipulation by skilled scientists but the performance of correct inferences about its target system. This requires, at a fundamental level, understanding how the model works, which is usually a matter of tinkering and testing hypothetical situations. As they put it, it is a matter of understanding the model, which consists in 'the abilities to manipulate the external inferential apparatus: in order to understand a model, one needs to understand how model properties change as a result of local changes in the assumptions' (ibid.). Understanding with the model then occurs when one successfully uses the model in empirical interventions and predictions. This is why the quality of a model can only be attested in hindsight, after its success has been confirmed in practice.

They further link their inferentialist notion of understanding with factivity. There is, however, an important distinction at play. Strictly speaking, factivity is a property of (true) propositions. But, as Kuorikoski and Ylikoski (ibid.) acknowledge, models are not sentential in structure, so their idea of factivity does not imply a semantic conception. Accordingly, their pragmatist idea of 'putting one's knowledge to use' through the performance of correct inferences involves only a weaker notion of factivity which amounts to pragmatic success. Thus, their inferentialist account of understanding provides only what they acknowledge to be a deflationary view on how models represent, according to which:

There is no substantial and general philosophical explanation for this representational success. These (perfectly objective) dependencies between the properties of external inferential apparatuses [such as a specific diagram, a set of equations, or a physical scale model] and their possible applications, i.e., the ways in which cognitive agents can perform inferential tasks with different kinds of external aids, are empirical and therefore proper objects of study for cognitive science, not philosophy. There are genuine philosophical puzzles, but the 
problem of representation is not one of them. (Kuorikoski \& Ylikoski. 2015).

So, if successful models are explained in terms of their pragmatic virtues, it is fair to ask why an inferentialist account of model-based cognition should commit to representationalism in the first place. We claim that the putative representational character of scientific models is superfluous in this kind of account - and, given the issues discussed in the previous subsection, avoiding representationalism is an advantage of our view.

To summarize: models are epistemic artifacts that scaffold scientific understanding about their target systems. This is only possible because models are materialized in various media, depending on the scientists' goals and the nature of their inquiry-with the possible exception of thought experiments, if these should be considered models at all. Scientific models allow us to gain understanding about their targets not by representing them, but by being manageable for skilled individuals. Therefore, whereas, in basic cognition according to EE, environmental structures afford possibilities of engagement for agents with certain bodily morphologies, sensorimotor abilities and goals; in scientific cognition, models afford possibilities of scientific engagements for agents with certain bodily morphologies, sensorimotor abilities and goals. By 'possibilities of scientific engagements' we understand the ability to intervene upon and to predict the behavior of a target system, which can be expresses as the performance of successful inferences. By 'agents with specific bodily morphologies and sensorimotor abilities' we understand humans of course - we cannot imagine in finer details how a truly alien science would look like. Importantly, this qualification also includes the scientist's training, her skills and her background. By 'goals' we mean naturally what the scientific inquiry in question aims to achieve. Different disciplines and different problems plausibly require different models and different kinds of engagements. In scientific cognition, as in basic cognition, individuals stablish and refine patterns of sensorimotor engagement with their immediate environment, that is, they dynamically enact rules for engagement. In the case of scientific cognition, sensorimotor engagements are constrained by how the models are materialized, and modeling activity is a dynamical process that is always open to refinement and the development of more productive and specialized tools. This is how scientists make sense, as enactivists put it, of their scientific environment: tinkering with models is a way to understand the empirical and conceptual problems they are dealing with, the phenomena under scrutiny and the data they gathered. 


\section{Material Engagement Theory and scientific cognition}

So far, we have seen how basic and scientific cognition share important aspects, even if more is needed in order to completely account for scientific cognition (and higher cognition in general). In this section, we explore how what is at play in model-based cognition is in fact continuous with basic cognition, for model manipulation is hereby understood as a special case of material engagement. In order to do so, we use material engagement theory to discuss cases of basic cognition (i.e., cognition about 'here and now physical structures') that involve artifacts, such as clay objects, stone tools, and so on.

\subsection{Materially engaged cognition}

Material engagement theory (henceforth, MET) was originally proposed as an alternative to the prevailing Cartesian dualism in the cognitive sciences and, consequently, in cognitive archeology as well (Malafouris, 2013). ${ }^{8}$ According to a Cartesian or cognitivist view, the mark of cognition is the symbolic processing of information as it occurs in the brain, independently of any external elements (Garofoli, 2016). Thus, cognitivist approaches to archeology take the cultural artifacts made by ancestral human beings and studied by archeologists as epiphenomena of those ancestors' cognitive processes (Garofoli, ibid.). MET explicitly challenges the Cartesian and cognitivist assumptions - thus being a natural ally for $\mathrm{EE}$ approaches - by suggesting that those artifacts were not projected by the minds of those ancestors, they are instead constitutive of their minds (Aydin, 2015).

Before expanding on MET's details, it is worthwhile to contrast it with extended cognition, a view that also broadens the limits of the mental beyond the brain, but does so less radically than MET. In general, extended cognition claims that cognitive processes may be temporally coupled with external devices (Clark \& Chalmers, 1998). For instance, utilizing agendas, notepads and calculators are typical ways of extending cognition. In these cases, external devices allow for cognitive offload, enabling the individual to focus on more complex tasks.

\footnotetext{
${ }^{8}$ Cartesian dualism ontologically divides the world in mental and physical parts. Mental is conceived as an internal category of phenomena, encapsulated in the thinking subject. Nowadays, the ontological aspect of Cartesian dualism is generally discharged in favor of physicalism - but traditional cognitive sciences still conceive of cognitive phenomena as internal phenomena encapsulated in subjects (or in their brains). The division between res cogitans and res extensa is thereby replaced by the division between cognitive processing and physical phenomena (Aston, 2019).
} 
Kuorikoski and Ylikoski (2015) explicitly base their account of modeling on extended cognition (models are external devices on their view), which may explain their sympathy for the representationalism of model-based cognition. This is so because extended cognition is in principle compatible with representationalism, for external devices would function as nonbiological vehicles of representational content for the organism. Representationalism, as we saw, is an important divergent ground between Kyorikoski and Ylikoski's account of understanding and ours.

Contrary to extended cognition, which conceives of cognition as centered on the brain with the possibility of being coupled to external devices; for MET, cognition is an ongoing, open-ended processes that is constituted in the enactive engagement between brain, body and environment, with emphasis on artifacts, tools and material cultures in general (Malafouris, 2013, 2019). In accordance with the EE approach, MET claims that cognition cannot be properly understood in absence of these elements. Therefore, cognition cannot be extended for it already involves the environment. In the cases of basic cognition that involve engaging with artifacts, cognition is not taken to be an abstract process that happens in the brain of an agent and is extended through external vehicles. Instead, cognition (in these cases) is the concrete manipulation of artifacts by an embodied agent. In order to explore this view, Malafouris coins the word "thinging" which 'incorporates time-varying and culture-specific bodily techniques; it also extends to sensory and cognitive prostheses and interfaces of any kind' and shifts our attention away from 'the sphere of isolated and fixed categories (objects, artefacts, etc.) to the sphere of the fluid and relational transactions between people and things' (Malafouris, 2014, p. 143). Casting these ideas in EE terms, we can say that cognition involving artifacts is the ongoing, open ended and dynamic manipulation of material cultures, which constitutively involves these artifacts, as well as sensorimotor engagements from embodied agents, selection of affordances and, perhaps more importantly, the creation of novel affordances too. ${ }^{9}$

\footnotetext{
${ }^{9}$ Perceiving one's surroundings through tools characterizes the Homo sapiens as a species. From to the first stone tools to more complex artifacts used to understand astronomic events (probably in order to assist in harvest), the human capacity to know is always materially mediated (Chakrabarty, 2019). Insofar as human knowledge takes place in virtue of the human capacity to act in the world through artifacts, Homo faber might be a more appropriate name than Homo Sapiens (Ihde \& Malafouris, 2019). Malafouris $(2013 ; 2019)$ illustrated the point with the classic example of the cane used by a blind person: a substantial part of her experience of reality is mediated by the cane, analogously to the way that a sighted person may use a glass. The cane thus becomes a constitutive part of the experience of being in the world. Brain, body and cane are coupled, and it is this coupling that constitutes the person's cognitive access to her environment. Therefore, the cyborg status that Clark ascribes to the human species has a more radical meaning according to MET (Chakrabarty, 2019; A Clark, 2003; Ihde \& Malafouris, 2019).
} 


\subsection{Numerosity and MET}

MET has consequences for human cognition that are not foreseen by other theories. This is due to the way it relates agency and the material constitution of artifacts (in contrast to functional theories, such as extended cognition and, more controversially, behaviorism-for a discussion, see Lazzeri, 2017). The cognitive consequences of the difference in the material constitution of artifacts can be seen in numeracy. Numeracy is the mathematical proficiency needed to perform calculations, from simpler to more complex ones. This more refined capacity is developed by being based on the more basic sensorimotor abilities of distinguishing quantities, which we share with other species. More precisely, numeracy takes place through the material engagements that amplify mathematical capacities, not because more basic abilities become enhanced through external devices, but because they form new couplings with artifacts whose physical properties afford different kinds of mathematical operations (i.e., thinking with words and numbers is in fact a matter of thinging, to use Malafouris's neologism) (Overmann, 2016b; Roepstorff, 2008).

The artifacts that improve our mathematical capacities and enable numeracy range from words to mathematical systems (Overmann, 2016b; Roepstorff, 2008). More specifically, words and numerical systems represent quantities that allow them to be manipulated more easily by being written in a given medium, or by being embodied in an object. Thus, concrete symbolization offers new affordances that are not in place in finger-counting, for instance. Sure, adding small quantities is easy with finger-counting, but it allows for fewer operations than utilizing strokes and marks in wood or clay (Overmann, 2013, 2016a, 2019; Overmann \& Wynn, 2019; Roepstorff, 2008). Consider a prisoner counting his days on prison. Finger-counting will not help if his sentence is longer than 20 days, and repeating fingers may in fact hinder the counting, because it adds a further task of keeping track of how many times each finger was used. This is not a problem if, instead of finger-counting, he marks the walls with a stone, in which each mark counts as a day. In this scenario, the prisoner has the whole room to count days, and he may as well create new symbols for weeks, months and years. In that case, the biological dispositions involved in cognition were not enhanced by external devices; instead, the use of technology, rudimentary as it is, produced a more elaborated way of cognizing, which is not reduced to the artificial components nor to the biological ones, but it is tailored to suit the embodied abilities and dispositions of the individual and is constrained by the material constitution of the medium. This is in tune with our construal of model-based cognition, where 
the technology that affords the "thinging" process are scientific models, which in turn can be materialized in the many ways we mention in section 2 .

Something similar happened when scribes, merchants, traders and farmers in Ancient Near Eastern civilizations began to use clay objects to help counting (Overmann, 2016a, 2016b). Circa 7000 B.C., clay was first used to create objects (tokens) in order to track kinds and quantities of commodities such as food. Later (3300 B.C.) these objects were used to impress hollow clay envelopes that contained the clay tokens. Envelopes were later replaced by solid clay tablets with the impression of those tokens representing the commodities. Eventually, impressing tokens on tablets was replaced by pictographs, which led to the creation of signs (phonograms) in around 3000 B.C. (for a detailed discussion, see Malafouris, 2013, chapter 5). Importantly, the possibility of modeling different quantities and kinds of objects with different materials allowed for the abstract notion of number, thus enabling more complex mathematical operations, plausibly in virtue of the fact that clay is a malleable material that affords more freedom in manipulation than other objects such as rocks. Similarly, geometry emerged from the processes of "thinging" (Gallagher, 2015), for objects with specific layouts, which were built with assorted materials and with pragmatic goals in mind (counting crops, keeping track of debts, etc.), probably served as bases for the abstract ideas of geometrical figures and their properties (Ferreirós \& García-Pérez, 2020).

The above discussion goes to show that, if the interpretation of modeling activity presented in the previous section is correct, then there is a continuity between what we have called basic cognition, when it involves material engagements, and scientific cognition. For MET describes what goes on in basic cognition involving artifacts as a coupling between agent and environment, a coupling that scaffolds new ways of cognizing. This is exactly what happens in model-based cognition, where models scaffold inferences about their target domain. Moreover, MET also has consequence for some cases of higher cognition. Even granting that higher cognition is sometimes a matter of engaging with abstract targets, as in mathematical operations, these procedures are phylogenetically and developmentally grounded on "thinging". Thus, at least in the cases where higher cognitive activity can be traced to the manipulation of artifacts, a performance of higher cognition is distributed through brain, body and material cultures in intricated and complex ways. Which is to say that at least some cases of higher cognition are based on sensorimotor engagements coupled with material artifacts, 
which have a rich history of socially shared use and offer specific affordances for skilled individuals. ${ }^{10}$

\section{Explaining away the gap between basic cognition and scientific cognition}

Before wrapping up the preceding discussions and exploring some of its consequences, let us summarize our itinerary so far. At the outset we presented the EE approach (insofar as an integrated approach between enactivism and ecological psychology is possible), which faces the challenge of explaining higher cognition. If EE fails to do so, an explanatory gap between basic and higher cognition ensues. Here we have focused on scientific cognition as a paradigmatic case of higher cognition in order to show that there is a continuum between basic and higher cognition. We supported that claim by arguing that scientific cognition is a matter of achieving understanding of how models work and of how the target system works. This is similar to how agents engage with their immediate, physical environment according to the EE approach. Models, we claimed, are always materialized, and it is their material embodiment that enables prolific interactions by trained individuals. These ideas are developed in a nonrepresentationalist manner, for models do not represent their targets (in fact, they are purposely designed to be unlike their targets), whereas understanding how is hereby construed as an ability to intervene upon and to predict the behavior of the target system through successful inferences. In section 3, we explored MET to show that material engagements, such as modelbased cognition, can be cast in EE terms, thus dissolving the temptation to project a gap in our cognitive spectrum.

In this paper, we argued that the artifacts that constitute our cognitive activity in scientific practice are designed to afford engagements according to the agents' bodily morphologies, sensorimotor abilities and research goals. Moreover, the process of creating these artifacts is ongoing and open-ended, and it leads to the refinement of the models in use, the development of new ones, and the emergence of new ways of engaging with them. To the extent that the

\footnotetext{
${ }^{10}$ From the point of view of the neurodynamics involved, MET offers a distinctive view on how material engagements shape our cognitive activity. Whereas a cognitivist would claim that processes that involve artifacts cause (over large time scales) more complex brain activity that leaves material traces as mere epiphenomena, MET takes material engagement as transformative of cognition. According to MET, agents (not brains alone, but embodied, situated brains) are engaged in activities with tools which are materialized in many ways, and these engagements in turn may reorganize neural activity and deeply affect the agent's sensorimotor abilities. Over sufficiently large time scales, couplings of that kind allow for new kinds of engagements and the development of new artifacts. Thus, the engagements with different technologies causes the reuse of brain areas that were developed for other tasks (Jones, 2018) — possibly the ones related to basic cognition.
} 
same goes on in basic cognition, there is a continuum between basic and higher cognition. Thus, when it comes to model-based cognition, models are not something new-for, despite being more salient in modern science, they are special kinds of artifacts, and their use draws upon other kinds of material interactions that have been established in previous engagements. ${ }^{11}$ Fundamentally, therefore, scientific procedures are deeply rooted in our embodied nature, and if model-based science is a paradigmatic case of higher cognition, then the EE approach is not liable to the charge of being gappy.

Finally, we want to highlight two further consequences of the views developed here. Firstly, we have refrained from mentioning scientific theories, for our focus is on models and the understanding they provide. But our take on scientific cognition may provide an interesting insight when it comes to theories. Traditionally, theories are taken to be highly abstract entities and, therefore, to be starkly departed from the kind of engagement characterized by modelbased cognition. However, theories are clearly susceptible to the outcomes of modeling engagements, for a theory not only guides how a model is built, but it may also be amended by how the model works in practice. If the two, model and theory, are kept radically apart, it is hard - if not impossible - to see how these mutual relations take place. Instead, MET suggests that a theory is not stored in the researcher's mind, which would be merely expressed in a given medium. Just like geometric ideas were only brought forth after concrete objects with the relevant properties were produced, the same may go on for theories and models (we tentatively speculate). Thus, even if theories have a higher degree of abstraction than models — and, as we emphasize at the outset, more than what the EE and MET offer may be needed to explain what makes a scientific theory a good one-in practice they are ultimately meshed with the scientist's modeling activity. Just like an ancestral human could not form a mental representation of a stone tool completely apart from the actions of using that tool (Chakrabarty,

\footnotetext{
11 Even though models are a special kind of artifacts (and material engagements happens at basic levels of cognition), this does not mean that modern day science could be developed in the same way in the remote past. Consider the epistemic and pragmatic consequences of using computers and data, which is spread across basically every scientific areas today, from physics to social sciences (Vallverdú i Segura, 2009). For instance, in mathematics, the proofs of many theorems are no longer elaborated on pen and paper, instead they are done by computers that are capable of performing more calculations in less time than a person (for some caveats on this issue, see Casacuberta \& Vallverdú, 2014). Similarly, astronomy, cosmology and physics require long and complex calculations that could only be accomplished by computers. In social sciences and in biology, large data sets are used to base conclusions about populations, which, without computers, would be impractical or highly time consuming in virtue of the amount of data and the complexity of the statistical analyses involved. So, if the material constitution of technology deeply influences our cognitive complexity, then certain scientific endeavors would be unfathomable for people of different ages. This means that even if a scribe from the Babylonian empire somehow were to learn present-day astronomy, he would be incapable to perform successfully in that area by using only clay elements, due to the lack of computers needed to manipulate a huge amount of data.
} 
2019; Ihde \& Malafouris, 2019), maybe theories could not be brought forth without certain kinds of engagements and possibilities for action.

Thus, instead of thinking of scientific theories as abstract entities, we may think of them instead as an ongoing activity, what we may call theorizing. We can retain the intuitive difference between model manipulation and theorizing of course, because theorizing involves something else than enaction, affordance selection and material engagements. It requires, for instance, proficiency in complex languages, formal rigor, sophisticated inferences and so on. But even so, theorizing takes place in practice, typically in material media. For instance, a scientist working in a lab takes notes in a board or in a notepad in order to avoid ambiguity and doubts about her research procedures, or in order to clarify what to do in the case of a problem that she has faced before-which is crucial for collaborative endeavors. This process works as a scaffolding, allowing for the researches to engage in other activities that may depend on the overcoming of those issues (from the perspective of extended mind, one could say that these instructions allow for the cognitive offload) (Goldsworthy, 2019). This is all part of the process of theorizing and it guides, as well as is influenced by, the practical success of modeling activity.

Secondly, and in relation to the previous point, our view may allow us to draw a more general picture in which laboratorial practice is construed as a basal level of scientific engagement. This can be improved, for instance, by following formal instructions and by conducting experiments, eventually scaling up to scientific understanding and the production of knowledge. The idea is that not only laboratorial techniques are first acquired in practice-for instance, as one uses scientific instruments in experimentations and becomes acquainted with how they work-, but also that higher levels of scientific cognition are dependent on socially transmitted skills and know-how. Learning to use a model, tinkering with it, and applying it in varying contexts may constitute intermediary degrees of scientific cognition that connect experimentation and theorizing, informing and being informed by both ends of this spectrum. This is a possibility we would like to explore in the future. ${ }^{12}$

\section{References}

\footnotetext{
${ }^{12}$ We thank an anonymous reviewer for this suggestion.
} 
Aston, A. (2019). Metaplasticity and the boundaries of social cognition: Exploring scalar transformations in social interaction and intersubjectivity. Phenomenology and the Cognitive Sciences, 18(1), 65-89. https://doi.org/10.1007/s11097-018-9601-z

Aydin, C. (2015). The artifactual mind: Overcoming the 'inside-outside' dualism in the extended mind thesis and recognizing the technological dimension of cognition. Phenomenology and the Cognitive Sciences, 14(1), 73-94. https://doi.org/10.1007/s11097-013-9319-X

Bailer-Jones, D. (2003). When Scientific Models Represent. International Studies in the Philosophy of Science, 17, 59-74.

Barandiaran, X. E. (2017). Autonomy and Enactivism: Towards a Theory of Sensorimotor Autonomous Agency. Topoi, 36(3), 409-430. https://doi.org/10.1007/s11245-016-93654

Bruineberg, J., Chemero, A., \& Rietveld, E. (2019). General ecological information supports engagement with affordances for 'higher' cognition. Synthese, 196(12), 5231-5251. https://doi.org/10.1007/s11229-018-1716-9

Carvalho, E. M. de, \& Rolla, G. (2020). An Enactive-Ecological Approach to Information and Uncertainty. Frontiers in Psychology, 11. https://doi.org/10.3389/fpsyg.2020.00588

Casacuberta, D., \& Vallverdú, J. (2014). E-Science and the data deluge. Philosophical Psychology, 27(1), 126-140. https://doi.org/10.1080/09515089.2013.827961

Chakrabarty, M. (2019). How stone tools shaped us: Post-phenomenology and material engagement theory. Philosophy \& Technology, 32(2), 243-264. https://doi.org/10.1007/s13347-018-0310-х

Chemero, A. (2009). Radical Embodied Cognitive Science. Cambridge, Massachusetts: The MIT Press.

Clark, A. (2003). Natural-born cyborgs: Minds, technologies, and the future of human. Oxford: Oxford University Press.

Clark, Andy. (2016). Surfing Uncertainty. Oxford: Oxford University Press.

Clark, Andy, \& Chalmers, D. (1998). The Extended Mind. Analysis, 58(1), 7-19.

Clark, Andy, \& Toribio, J. (1994). Doing without representing? Synthese, 101(401-431). de Oliveira, G. S. (2018). Representationalism is a dead end. Synthese. https://doi.org/10.1007/s11229-018-01995-9

Di Paolo, E. A. (2005). Autopoiesis, Adaptivity, Teleology, Agency. Phenomenology and the Cognitive Sciences, 4(4), 429-452. https://doi.org/10.1007/s11097-005-9002-y

Di Paolo, E., Burhmann, T., \& Barandiaram, X. (2017). Sensorimotor Life: An Enactive 
Proposal. Oxford, New York: Oxford University Press. https://doi.org/10.1093/acprof:oso/9780198786849.001.0001

Di Paolo, E., Cuffari, E. C., \& De Jaegher, H. (2018). Linguistic Bodies: The Continuity Between Life and Language. Cambridge, Massachusetts: MIT Press.

Elgin, C. (2007). Understanding and the facts. Philosophical Studies, 132(1), 33-42. https://doi.org/10.1007/s11098-006-9054-Z

Elgin, C. (2009). Is Understanding Factive? In D. Pritchard, A. Millar, \& A. Haddock (Eds.), Epistemic Value (pp. 322-330). Oxford: Oxford University Press.

Ferreirós, J., \& García-Pérez, M. J. (2020). Beyond natural geometry: On the nature of protogeometry. Philosophical Psychology, 33(2), 181-205. https://doi.org/10.1080/09515089.2019.1683726

Fraassen, B. van. (1980). The Scientific Image. Oxford: Clarendon Press.

Frigg, R., \& Hartmann, S. (2020). Models in Science. In E. N. Zalta (Ed.), The Stanford Encyclopedia of Philosophy (Spring 202). Metaphysics Research Lab, Stanford University.

Frigg, R., \& Nguyen, J. (2017). Models and Representation. In L. Magnani \& T. Bertolotti (Eds.), Springer Handbook of Model-Based Science. Cham: Springer International Publishing. https://doi.org/10.1007/978-3-319-30526-4

Gallagher, S. (2015). Doing the math: Calculating the role of evolution and enculturation in the origins of geometrical and mathematical reasoning. Progress in Biophysics and Molecular Biology, 119(3), 341-346. https://doi.org/10.1016/j.pbiomolbio.2015.06.016

Garofoli, D. (2016). Cognitive archaeology without behavioral modernity: An eliminativist attempt. Quaternary International, 405, 125-135.

Gelfert, A. (2017). The Ontology of Models. In L. Magnani \& T. Bertolotti (Eds.), Springer Handbook of Model-Based Science. Cham: Springer International Publishing. https://doi.org/10.1007/978-3-319-30526-4

Gibson, J. J. (2015). The Ecological Approach to Visual Perception. New York: Psychology Press.

Giere, R. N. (1988). Explaining Science: a Cognitive Approach. Chicago: University of Chicago Press.

Giere, R. N. (2004). How Models Are Used to Represent Physical Reality. Philosophy of Science, 71, 742-752.

Goldsworthy, C. (2019). The effect of dynamic social material conditions on cognition in the biomedical research laboratory. Phenomenology and the Cognitive Sciences, 18(1), 241- 
257. https://doi.org/10.1007/s11097-018-9600-0

Heft, H. (2020). Ecological Psychology and Enaction Theory: Divergent Groundings.

Frontiers in Psychology, 11. https://doi.org/10.3389/fpsyg.2020.00991

Heras-Escribano, M. (2019). Pragmatism, enactivism, and ecological psychology: towards a unified approach to post-cognitivism. Synthese, (0123456789).

https://doi.org/10.1007/s11229-019-02111-1

Hills, A. (2009). Moral Testimony and Moral Epistemology. Ethics, 120, 94-127.

Hutto, D. D., \& Myin, E. (2013). Radicalizing Enactivism: Basic Minds without Content. Cambridge, Massachusetts: MIT Press.

Hutto, D. D., \& Myin, E. (2017). Evolving Enactivism: Basic Minds Meet Content. Cambridge, Massachusetts: The MIT Press.

Hutto, D. D., \& Satne, G. (2015). The Natural Origins of Content. Philosophia, 43(3), 521536.

Ihde, D., \& Malafouris, L. (2019). Homo faber revisited: Postphenomenology and material engagement theory. Philosophy \& Technology, 32(2), 195-214. https://doi.org/10.1007/s13347-018-0321-7

Jones, M. (2018). Numerals and neural reuse. Synthese, 1-25. https://doi.org/10.1007/s11229-018-01990-0

Kiverstein, J. D., \& Rietveld, E. (2018). Reconceiving representation-hungry cognition: an ecological-enactive proposal. Adaptive Behavior, 26(4), 147-163. https://doi.org/10.1177/1059712318772778

Knuuttila, T. (2005). Models, Representation, and Mediation. Philosophy of Science, 72(5), 1260-1271. https://doi.org/10.1086/508124

Knuuttila, T., \& Merz, M. (2009). Understanding by Modeling: an Objectual Approach. In H. de Regt, S. Leonelli, \& K. Eigner (Eds.), Scientific Understanding. Pittsburgh: University of Pittsburgh Press.

Knuuttila, T., \& Voutilainen, A. (2003). A Parser as an Epistemic Artifact: A Material View on Models. Philosophy of Science, 70(5), 1484-1495. https://doi.org/10.1086/377424

Kuorikoski, J., \& Ylikoski, P. (2015). External representations and scientific understanding. Synthese, 192(12), 3817-3837. https://doi.org/10.1007/s11229-014-0591-2

Lazzeri, F. (2017). Extended functionalism from a behavioral perspective. Behavior and Philosophy (Online), 45, 1-21.

Leonelli, S. (2009). Understanding in Biology: The Impure Nature of Biological Knowledge. In H. de Regt, S. Leonelli, \& K. Eigner (Eds.), Scientific Understanding. Pittsburgh: 
University of Pittsburgh Press.

Lipton, P. (2009). Understanding Without Explanation. In H. Regt, S. Leonelli, \& K. Eigner

(Eds.), Scientific Understanding. Pittsburgh: University of Pittsburgh Press.

Magnani, L. (2017). The Abductive Structure of Scientific Creativity (Vol. 37). Cham:

Springer International Publishing. https://doi.org/10.1007/978-3-319-59256-5

Malafouris, L. (2013). How things shape the mind: A Theory of Material Engagement.

Cambridge, Massachusetts: MIT Press.

Malafouris, L. (2014). Creative thinging. Pragmatics \& Cognition, 22(1), 140-158.

https://doi.org/10.1075/pc.22.1.08mal

Malafouris, L. (2019). Mind and material engagement. Phenomenology and the Cognitive

Sciences, 18(1), 1-17. https://doi.org/10.1007/s11097-018-9606-7

Moyal-Sharrock, D. (2019). From deed to word: gapless and kink-free enactivism. Synthese.

https://doi.org/10.1007/s11229-019-02218-5

Novaes, F. C. (2020). O dualismo vestigial: Os caminhos da mente estendida e engajamento material para a continuidade mente-matéria. Cognitio-Estudos: Revista Eletrônica de Filosofia, 17(1), 92-101. https://doi.org/10.23925/1809-8428.2020v17i1p92-101

Overmann, K. A. (2013). Material scaffolds in numbers and time. Cambridge Archaeological Journal, 23(1), 19.

Overmann, K. A. (2016a). Beyond writing: The development of literacy in the Ancient Near East. Cambridge Archaeological Journal, 26(2), 285-303.

https://doi.org/10.1017/S0959774316000019

Overmann, K. A. (2016b). The role of materiality in numerical cognition. Quaternary International, 405, 42-51. https://doi.org/10.1016/j.quaint.2015.05.026

Overmann, K. A. (2019). Concepts and how they get that way. Phenomenology and the Cognitive Sciences, 18(1), 153-168. https://doi.org/10.1007/s11097-017-9545-8

Overmann, K. A., \& Wynn, T. (2019). Materiality and human cognition. Journal of Archaeological Method and Theory, 26(2), 457-478. https://doi.org/10.1007/s10816018-9378-y

Pincock, C. (2012). Mathematics and Scientific Representation. Oxford: Oxford University Press.

Pritchard, D. (2008). Knowing the Answer, Understanding and Epistemic Value. Grazer Philosophische Studien, 77, 325-339.

Regt, Henk de. (2009). Understanding and Scientific Explanation. In Henk de Regt, S. Leonelli, \& K. Eigner (Eds.), Scientific Understanding. Pittsburgh: University of 
Pittsburgh Press.

Regt, Henk de, Leonelli, S., \& Eigner, K. (2009). Focusing on Scientific Understanding. In

Henk de Regt, S. Leonelli, \& K. Eigner (Eds.), Scientific Understanding. Pittsburgh:

University of Pittsburgh Press.

Rietveld, E., \& Kiverstein, J. (2014). A Rich Landscape of Affordances. Ecological

Psychology, 26(4), 325-352. https://doi.org/10.1080/10407413.2014.958035

Roepstorff, A. (2008). Things to think with: Words and objects as material symbols.

Philosophical Transactions of the Royal Society B: Biological Sciences, 363(1499),

2049-2054. https://doi.org/10.1098/rstb.2008.0015

Rowlands, M. (2017). Arguing about Representations. Synthese, 194(11), 4215-4232.

Segundo-Ortin, M. (2020). Agency From a Radical Embodied Standpoint: An Ecological-

Enactive Proposal. Frontiers in Psychology, 11(June), 1-13.

https://doi.org/10.3389/fpsyg.2020.01319

Suárez, M. (2003). Scientific Representations: Against Similarity and Isomorphism.

International Studies in the Philosophy of Science, 71, 767-779.

Turvey, M. T., Shaw, R. E., Reed, E. S., \& Mace, W. M. (1981). Ecological laws of

perceiving and acting: In reply to Fodor and Pylyshyn (1981). Cognition, 9(3), 237-304.

https://doi.org/10.1016/0010-0277(81)90002-0

Vallverdú i Segura, J. (2009). Computational Epistemology and e-Science: A New Way of

Thinking. Minds and Machines, 19(4), 557-567. https://doi.org/10.1007/s11023-009-

9168-0

van den Herik, J. C. (2018). Attentional actions- A n ecological-enactive account of

utterances of concrete words. Psychology of Language and Communication, 22(1), 90-

123. https://doi.org/10.2478/plc-2018-0005

van den Herik, J. C. (2020). Rules as Resources: An Ecological-Enactive Perspective on

Linguistic Normativity. Phenomenology and the Cognitive Sciences.

https://doi.org/10.1007/s11097-020-09676-0

Ward, D., Silverman, D., \& Villalobos, M. (2017). Introduction: The Varieties of Enactivism.

Topoi, 36(3), 365-375. https://doi.org/10.1007/s11245-017-9484-6

Winsberg, E. (1999). Sanctioning Models: The Epistemology of Simulation. Science in

Context, 12(2), 275-292. https://doi.org/10.1017/S0269889700003422 